\title{
SARS-COV-2 VACCINE IN SPONDYLOARTHRITIS PATIENTS: OVERALL MODERATE/HIGH IMMUNOGENICITY IMPAIRED BY IMMUNOSUPPRESSANTS AND BIOLOGICAL THERAPY
}

Carla Gonçalves Schahin Saad ${ }^{1}$, Matheus Santos Rodrigues Silva ${ }^{1, \star}$, Percival Degrava Sampaio Barros ${ }^{1}$, Julio Cesar Bertacini de Moraes ${ }^{1}$, Cláudia Goldenstein Schainberg ${ }^{1}$, Nadia Emi Aikawa ${ }^{1}$, Emily Figueiredo Neves Yuki ${ }^{1}$, Sandra Gofinet Pasoto ${ }^{1}$, Tatiana do Nascimento Pedrosa ${ }^{1}$, Renato Kenji Aoyama ${ }^{1}$, Carlo Scognamiglio Renner Araujo ${ }^{1}$, Clóvis Artur Almeida da Silva ${ }^{1}$, Ana Cristina de Medeiros Ribeiro ${ }^{1}$, Eloisa Silva Dutra de Oliveira Bonfá ${ }^{1}$

1.Universidade de São Paulo, São Paulo (SP), Brazil.

*Corresponding author: matheussilva66@yahoo.com.br

\section{BACKGROUND}

We recently reported an attenuate immunogenicity in patients with autoimmune rheumatic diseases. However, the effect of spondyloarthritis (SpA) and its treatment on COVID-19 vaccine immunogenicity remains to be determined for this group of patients. We therefore aimed to evaluate humoral immune responses to inactivated SARS-CoV-2 vaccine (CoronaVac) in patients with SpA (axial spondyloarthritis and psoriatic arthritis) taking DMARDs and commonly used targeted biological therapies, compared with a control group (CG).

\section{METHODS}

Prospective observational cohort patients diagnosed with 194 SpA and 183 CG were vaccinated with CoronaVac in two doses with a 28-days interval. One hundred ninety-four patients completed the study and could be paired with CG for immunogenicity analysis. Blood samples were collected in the days 0, 28 and 69 (D69) to evaluate anti-SARS-CoV-2 IgG seroconversion (SC) and presence of neutralizing antibodies (NAb) in participants with negative $\lg G$ and NAb at baseline.

\section{RESULTS}

Patients and GC were comparable regarding age $(p=0.93)$ and sex $(p=1.00)$. Immunogenicity at D69 showed a moderate/high SC $(80.2 \%$ vs. $95.7 \%, p<0.0001)$ and NAb positivity $(61.6 \%$ vs. $82.7 \%, p<0.0001)$ in SpA but lower than CG. Factors associated with lower immunogenicity were older age ( 56.8 vs. $51.4 ; p=0.03318)$ and higher frequencies of prednisone $(25.7 \%$ vs. $4.2 \%$; $p=0.0004)$, methotrexate ( $51.4 \%$ vs. $40.1 \%, p=0.0016)$ and TNF inhibitor (TNFi) (62.9\% vs. 34.5\%, $p=0.0035)$. Likewise, prednisone $(17.6 \%$ vs. $2.8 \%, p=0.0013)$ and TNFi ( $50 \%$ vs. $33.9 \% ; p=0.0408)$ were associated with diminished NAb positivity. Sulfasalazine was associated with higher SC rates ( $8.6 \%$ vs. $26.8 \%, p=0.0246)$ and NAb positivity ( $13.2 \%$ vs. $29.4 \%, p=0.0168)$. The multivariate analysis revealed that older age ( $p=0.037)$, prednisone $(p=0.001)$, TNFi $(p=0.016)$, and methotrexate $(p=0.017)$ were independently associated with lower SC while prednisone $(p=0.006)$ and TNFi $(p=0.027)$ were also associated with reduced NAb response.

\section{CONCLUSION}

Our finding of an excellent safety and moderate/high SC rate in SpA supports the recommendation of CoronaVac vaccination. The impaired immune response in the minority of patients under immunosuppressive and biological therapy requires novel strategies to enhance antibody response in this subgroup of patients.

\section{KEYWORDS}

Spondyloarthritis, Vaccine, CoronaVac, COVID-19, Immunization. 